\title{
Estimation of Fluoride and Sirtuin1 in Patients with Diabetic Nephropathy in Kolar District of Karnataka, India
}

\author{
Sai Deepika Ram Mohan ${ }^{10} \quad$ Kurpad N. Shashidhar $^{1}$ \\ ${ }^{1}$ Department of Biochemistry, Sri Devaraj Urs Academy of Higher \\ Education and Research, Kolar, Karnataka, India \\ 2 Department of General Medicine, Sri Devaraj Urs Medical College, \\ Tamaka, Kolar, Karnataka, India \\ ${ }^{3}$ Department of Community Medicine, Sri Devaraj Urs Medical \\ College, Tamaka, Kolar, Karnataka, India \\ J Lab Physicians 2022;14:57-64.
}

\author{
Raveesha Anjanappa ${ }^{2}$ Muninarayana Chandrappa ${ }^{3}$ \\ Address for correspondence Kurpad N. Shashidhar, MD, Department \\ of Biochemistry, Sri Devaraj Urs Medical College, Tamaka 563103, \\ Kolar, Karnataka, India (e-mail: drshahsikn1971@yahoo.co.in).
}

\begin{abstract}
Keywords

- carboxymethyl lysine

- fluoride

- sirtuin 1

- therapeutics

- type-2 diabetes mellitus
\end{abstract}

Objective Prevalence of type-2 diabetes mellitus (DM) and diabetic nephropathy is growing rapidly in Asian countries, affecting low- and middle-income groups. One of the epidemiological issues of Kolar district is fluorosis; advanced glycation end product, carboxymethyl lysine (CML), and a molecule of interest Sirtuin 1 are employed in the present study. In the correlation of fluoride with sirtuin 1 and CML with sirtuin 1 of cases lies the important rationale of the study to assess the extent of kidney damage.

Materials and Methods This is a comparative cross-sectional study with three groups, each with 70 patients, as follows: G1, control; G2, diabetes with diabetic nephropathy; and G3, type-2 DM without any complications. Informed written consent was obtained from all study patients. All the routine investigations were performed by fully automated Vitro 5, $1 \mathrm{Fs}$, Vitros. Fasting insulin was analyzed by Vitro eCl and glycated hemoglobin was estimated by BioRad D10. Sirtuin1, CML, and fructosamine were estimated by double antibody sandwich technique.

Statistical Analysis The statistical analysis was performed by SPSS 20 (IBM) software. Means of normally distributed data were compared using analysis of variance (ANOVA), and not normally distributed data were compared by Kruskal-Wallis test. A p-value of less than 0.05 was considered statistically significant.

Results A decrease in sirtuin 1, serum, and urine fluoride of group 2 (34.74 [25.08-53.2], 0.24 [0.2-0.5], and 0.24 [0.16-0.41]) was observed compared with other groups. Increased CML and fluoride act as prooxidant, restricting the effect of sirtuin 1 on cellular damage, causing further complications such as increased insulin resistance and decreased insulin sensitivity.

Conclusion The alterations in serum sirtuin 1 levels indicate the severity of damage due to stress during hyperglycemia and fluoride toxicity; hence, sirtuin 1 can be considered as biomarker of aging. Subsequently, the correlation of CML, estimated glomerular filtration rate (eGFR), and fluoride with sirtuin 1 indicates that increasing sirtuin 1 may defend the forthcoming damage and could be considered in therapeutics. published online August 21, 2021
DOI https://doi.org/

$10.1055 / \mathrm{s}-0041-1732817$. ISSN 0974-2727.
(C) 2021. The Indian Association of Laboratory Physicians. All rights reserved.

This is an open access article published by Thieme under the terms of the Creative Commons Attribution-NonDerivative-NonCommercial-License, permitting copying and reproduction so long as the original work is given appropriate credit. Contents may not be used for commercial purposes, or adapted, remixed, transformed or built upon. (https://creativecommons.org/ licenses/by-nc-nd/4.0/)

Thieme Medical and Scientific Publishers Pvt. Ltd., A-12, 2nd Floor, Sector 2, Noida-201301 UP, India 


\section{Introduction}

Diabetes mellitus (DM) is a clinical syndrome characterized by unexplained weight loss, polydipsia, polyuria, polyphagia, nonhealing or delayed healing of wounds, and blurring of vision. The biochemical markers include fasting plasma glucose of $\geq 126 \mathrm{mg} / \mathrm{dL}$ and postprandial plasma glucose of $\geq$ $140 \mathrm{mg} / \mathrm{dL} .{ }^{1}$ The World Health Organization (WHO) prediction states that by 2030, DM may be the seventh leading cause of death worldwide. ${ }^{2}$ Prevalence of DM is growing rapidly in Asian countries affecting low- and middle-income groups. ${ }^{3}$ Mathers and Loncar, in 2006, stated that approximately 328 million people will be affected by DM, globally. ${ }^{2}$ Studies conducted by Verma et al, in 2012, showed India as the capital for DM with 40.9 million diabetic patients. ${ }^{4}$ An in-house study in 2010 demonstrated approximately 10\% prevalence of diabetes in local population. ${ }^{5}$

There are several factors influencing prevalence of DM such as heredity, environmental factors, and lifestyle modifications. Among these factors, environmental factor and lifestyle modifications are considered in this study. Environmental factor is described as chronic fluoride exposure in the study area and study population as studied by Verma et al. ${ }^{6}$ Under lifestyle modifications, there are various aspects such as sedentary lifestyle, unhealthy diet, improper knowledge regarding health checkup, and others, among which unhealthy diet is considered as one of the risk factors in this study that is assessed by serum carboxymethyl lysine (CML) levels.

CML is an advanced glycation end product (AGE) whose serum levels are mainly dependent on dietary intake. ${ }^{7}$ These factors increase oxidative stress in the body-generating reactive oxygen species (ROS). ${ }^{8}$ To combat the effects of fluoride and CML, either there must be antioxidants reserve in body or continuous activation of oxidants scavenging mechanisms must happen. ${ }^{9}$ There is need of a molecule which shall decrease and/or nullify the effects of both CML and fluoride. The molecule identified is Sirtuin 1 which is involved in longevity guarding cells from aging preventing oxidative damage. ${ }^{9}$ Sirtuin 1 , a nicotinamide adeninde dinucleotide (NAD)+-dependent deacetylase homologous to silent information regulator 2 gene (Sir2) of Saccharomyces cerevisiae (Baker's yeast). ${ }^{8,9}$ Ultimately, further molecular research on sirtuin 1 may fetch details regarding its exact pathways involved, resulting in therapeutic usage of sirtuin 1 in aging disorder, since it is considered as antiaging protein.

Diabetic nephropathy, a consequence of type- $2 \mathrm{DM}$, is increasing across the nations and higher in Asian Indians with a range of 30 to $40 \%$, as reported in the year 2015 , can be due to environmental and genetic factors. ${ }^{10}$ Since this study includes diabetic nephropathy, the association of CML and increased rigidity of filtering apparatus are proved to be deteriorating renal health by many human and animal studies. $^{11,12}$

Since fluoride concentration is increasing day by day in drinking water of Kolar district, along with incidence of type$2 \mathrm{DM}$ and other aging disorders, this study was planned as a preliminary trial. In this population, no studies have shown a correlation of fluoride with insulin and diabetes. The framed hypothesis is fluoride and CML may be an enhancer molecule of diabetes and sirtuin 1 shall be the molecule for counter action for fluoride toxicity. Estimation of sirtuin 1 levels in blood may give a clue to assess the damage by hyperglycemia collectively due to all kinds of causative factors of type-2 DM. Sirtuin 1 can be included as biomarker of aging and therapeutics in preventing factors accelerating aging.

\section{Materials and Methods}

This is a comparative cross-sectional study with three groups.

\section{Subjects}

Type-2 diabetic patients attending outpatient department (OPD), department of general medicine and diabetology, were recruited for the study after confirming inclusion and exclusion criteria. All the patients were residents of Kolar district for minimum of 3 years. Informed written consent was obtained from all study patients. Age- and gendermatched nondiabetics and healthy patients of same area were included as controls.

\section{Study Groups}

Group I ( $n=70)$ : age and gender matched healthy controls living in Kolar as the patient of the other groups.

Group II $(n=70)$ : type-2 DM with diabetic nephropathy. Group III $(n=70)$ : type-2 DM diabetic nephropathy.

\section{Inclusion Criteria}

Patients clinically proven to be type-2 DM with or without diabetic nephropathy and living and surviving in the same environment exposed to fluoride were included in this study.

\section{Exclusion Criteria}

Exclusion criteria are as follows: (1) patients with DM not living in Kolar and not exposed to fluoride, (2) patients taking drugs or other factors known to cause diabetes and/or diabetic nephropathy, (3) patients undergoing any type of dialysis, (4) acute kidney injury due to any cause and other renal pathologies, and (5) patients with other type of diabetes. SPSS version 20 (IBM) was used to perform statistical analysis. All the variables that are normally distributed (parametric), represented as mean \pm standard deviation (SD) and those which are nonparametric, represented as median (25th-75th percentile). Analysis of variance (ANOVA) was used for calculating probability ( $p$-value). Pearson's correlation $(r)$ and spearman's correlation were used to find the trend between two variables of normally and not normally distributed parameters, respectively. K-independent sample test was used to derive the $p$-value of nonparametric data and considered Kruskal-Wallis test for calculation. The chi-square test represents the significance of parameters between groups of not normally distributed data. A $p$-value of $<0.05$ was considered as statistically significant. Normal distribution was assessed by considering Kolmogorov-Smirnov significance values. 


\section{Sample Collection}

With all, strict aseptic precautions, making the patient lie in a comfortable position, 8-hour 4-mL fasting blood sample and 2-mL 2-hour postprandial blood sample was collected. Fasting sample was split into parts with specified sample requirement as serum (plain tubes), whole blood (HbA1c\%), and plasma (ethylenediamine tetraacetic acid [EDTA]) for parameters as mentioned in measurement methods. Corresponding urine sample was also collected from the study patients for urine fluoride analysis. Quality assurance was performed as per the criteria laid down in the Clinical Diagnostic Laboratory Services of college attached hospital facility and confirmed.

\section{Methodology}

All the routine investigations were performed by fully automated Vitro 5, 1 Fs, Vitros (Ortho Clinical Diagnostics, United States), fasting insulin was analyzed by Vitro eCI (Ortho Clinical Diagnostics, United States), and glycated hemoglobin was estimated by BioRad D10 (California, United States) based on the principle of high performance liquid chromatography (HPLC) at Biochemistry section of the Central Diagnostic Laboratory Services facility at attached hospital. Manual methods were performed at department of biochemistry of Sri Devaraj Urs Medical College. Blood pressure was measured before fasting blood collection by mercury sphygmomanometer, height was measured by manual stadiometer and, weight was recorded from digital weighing machine to calculate body mass index (BMI) as $\mathrm{kg} / \mathrm{m}^{2}$.

Sirtuin1, CML, and fructosamine were estimated by double antibody sandwich technique, measured at $\lambda \max =450$ $\mathrm{nm}$ and expressed in $\mathrm{ng} / \mathrm{mL}$ procured from Sincere biotech, China. ${ }^{13-15}$ Cystatin C (Cys C) was, measured by newly designed Agappe mispa i2 nephelometry based desktop instrument where intensity of the color is measured at $\lambda \max =650 \mathrm{~nm}$ expressed in $\mathrm{mg} / \mathrm{L}^{16}$ Serum and urine fluoride was analyzed by Orion Thermo Scientific Fluoride Ion Selective Electrode (ISE), United States. Readily available standards procured from thermo scientific with total ionic strength-adjusting buffer (TISAB) were used for analysis. Then, 1, 2, and $10 \mathrm{ppm}$ standards with TISAB II buffer were used for analyzing serum and urine fluoride.

Very low density lipoprotein (VLDL) calculated by dividing triglyceride (TG) by $5:^{17}$

$\mathrm{VLDL}=\mathrm{TG} / 5$

Non-high density lipoprotein (nHDL) is now gaining importance against VLDL and HDL, since it also has major role in assessing lipid accumulation in vascular diseases. ${ }^{18}$

non-HDL cholestrol = total cholesterol - HDL

Insulin resistance and sensitivity are calculated to get a glimpse of functioning of target cells against secreted insulin. Based on homeostasis model assessment (HOMA) and quantitative insulin-sensitivity check index (QUICKI) values, treatment and administration of insulin dosage may be decided since they are calculated using fasting glucose and insulin. In addition to diabetes, other disorders can also be staged by their values such as, obesity, hyperlipidemia, polycystic ovarian disease, and others. Therefore we consid- ered to calculate HOMA-insulin resistance (IR) $)^{19}$ and QUICKI by using the following formulae:

HOMA-IR: HOMA - IR $=($ fasting plasma insulin $\times$ fasting blood sugar) $/ 405)^{20}$

QUICKI: QUICKI = $1 /[\log ($ insulin $[\mu \mathrm{U} / \mathrm{mL}])+\log ($ glucose $[\mathrm{mg} / \mathrm{dL}])$

All the calculations are done considering their limitations.

\section{Results}

Demographic data and diabetic profile are normally distributed and represented as mean $\pm S D$ are tabulated in -Table 1. Patients recruited for this study are age and gender matched and hence they are not significant. Group 1 consisted of 31 (44.3\%) male, group 2 contained 47 (67\%) male, and group 3 with 37 (52.8\%) male patients of $p$-value of 0.08 , indicating that gender-matched patients were recruited for all the groups. In basic renal and lipid profile, all the parameters were significant across groups except non-HDL which had a broad reference range.

Advanced biomarkers of diabetic nephropathy (DN) and fluorosis, namely, serum sirtuin 1 , serum fluoride, urine fluoride, and CML are emphasized in - Table 2. Advance biomarkers of DN and fluorosis have broad range of detection and were skewed in distribution. The estimated variables in view of skewness were considered as nonparametric and represented them as median (25th-75th percentile). Reference ranges for the parameters are yet to be defined after population or multicentric study across the globe.

Due to broad range of values of short-term glycemic control, insulin estimation and calculation of diabetes indices, these are considered as nonnormally distributed and represented as median (25th-75th percentile). The results are shown in -Table 3. Except fasting insulin, all the parameters showed a significant difference in their values. Yet again, there is a vital increase in values of in group 3 but decrease in QUICKI giving a concern to prevent diabetic complication in future.

To support the findings derived from our study, we analyzed Cys $\mathrm{C}$ and calculated estimated glomerular filtration rate (eGFR) 2009 and 2012, considering both creatinine and creatinine with Cys $\mathrm{C}$, respectively. The values are documented in - Table 4.

Sirtuin 1 is known to get fluctuated both physiologically and pathologically. This made us to find the extent of fluctuation in comparison with serum CML and serum fluoride for which we correlated using Spearman's rho correlation. The values are tabulated in - Table $\mathbf{5}$.

To correlate the calculated values between groups 1 to 3 between HOMA-IR, QUICKI, and sirtuin1, we applied Spearman's rho correlation tool. The values are depicted in - Table 6 .

\section{Discussion}

As already mentioned, oxidative stress, insulin resistance, and ecological imbalances are the common reasons for metabolic disorder which must be battled by either inducing or activating a cascade of processes which may mask 
60 Sirtuin1 in Fluorosis and Diabetes Mellitus Sai Deepika et al.

Table 1 Demographic and biochemical parameters of all three groups

\begin{tabular}{|c|c|c|c|c|}
\hline Parameter & Group $1(n=70)$ & Group $2(n=70)$ & Group $3(n=70)$ & $p$-Value \\
\hline \multicolumn{5}{|l|}{ Demographic data } \\
\hline Age $(y)$ & $42.71 \pm 9.2$ & $56.04 \pm 8.2$ & $53.1 \pm 8.2$ & $<0.001$ \\
\hline $\mathrm{SBP}(\mathrm{mm} \mathrm{Hg})$ & $122.1 \pm 5.4$ & $137.6 \pm 17.6$ & $125.3 \pm 11.02$ & $<0.001$ \\
\hline $\mathrm{DBP}(\mathrm{mm} \mathrm{Hg})$ & $78.3 \pm 4.6$ & $87.53 \pm 11.2$ & $82.23 \pm 7.64$ & $<0.001$ \\
\hline BMI $\left(\mathrm{kg} / \mathrm{m}^{2}\right)$ & $24.1 \pm 3$ & $22.6 \pm 1.6$ & $23.2 \pm 1.83$ & $<0.001$ \\
\hline \multicolumn{5}{|l|}{ Basic diabetic profile } \\
\hline $\mathrm{FBS}(\mathrm{mg} / \mathrm{dL})$ & $93.94 \pm 9.4$ & $173.6 \pm 64.02$ & $182.3 \pm 67.7$ & $<0.001$ \\
\hline PPBS (mg/dL) & $115.1 \pm 16.2$ & $271.2 \pm 91$ & $273.1 \pm 103$ & $<0.001$ \\
\hline HbA1c (\%) & $5.5 \pm 0.5$ & $8.2 \pm 2$ & $9.2 \pm 2.4$ & $<0.001$ \\
\hline \multicolumn{5}{|l|}{ Basic renal profile } \\
\hline Blood urea (mg/dL) & $19.6 \pm 6.6$ & $69.03 \pm 27.5$ & $27.6 \pm 13.4$ & $<0.001$ \\
\hline Serum creatinine $(\mathrm{mg} / \mathrm{dL})$ & $0.66 \pm 0.1$ & $3.4 \pm 1.5$ & $0.68 \pm 0.21$ & $<0.001$ \\
\hline Uric acid $(\mathrm{mg} / \mathrm{dL})$ & $4.4(3.7-5.8)$ & $4.0(2.6-5.6)$ & $4.0(3.07-5.1)$ & 0.023 \\
\hline Serum albumin (g/dL) & $4.05 \pm 0.4$ & $2.8 \pm 0.8$ & $4.3 \pm 0.9$ & $<0.001$ \\
\hline Sodium (mEq/L) & $137 \pm 2.1$ & $133.5 \pm 5.1$ & $135.8 \pm 3.2$ & $<0.001$ \\
\hline Potassium (mEq/L) & $4.3 \pm 0.41$ & $4.6 \pm 0.9$ & $4.4 \pm 0.5$ & 0.016 \\
\hline \multicolumn{5}{|l|}{ Lipid profile } \\
\hline Total cholesterol $(\mathrm{mg} / \mathrm{dL})$ & $171.4 \pm 39.1$ & $157.6 \pm 59.6$ & $183.3 \pm 59.6$ & 0.007 \\
\hline Triglycerides (mg/dL) & $141(93-193.5)$ & $148(114.5-211)$ & $184(119.5-215.5)$ & 0.033 \\
\hline $\mathrm{HDL}(\mathrm{mg} / \mathrm{dL})$ & $39.3 \pm 10.1$ & $28.3 \pm 10.3$ & $40.9 \pm 13.7$ & $<0.001$ \\
\hline $\mathrm{LDL}(\mathrm{mg} / \mathrm{dL})$ & $98(76.2-122)$ & $87(50.7-110.5)$ & $104(86.8-127.3)$ & 0.01 \\
\hline $\mathrm{VLDL}(\mathrm{mg} / \mathrm{dL})$ & $28(17.7-36.4)$ & $30(23-42.2)$ & $36.9(24-43.1)$ & 0.020 \\
\hline $\mathrm{nHDL}(\mathrm{mg} / \mathrm{dL})$ & $132.1 \pm 39.5$ & $125.7 \pm 58.9$ & $145.4 \pm 56.8$ & 0.077 \\
\hline
\end{tabular}

Abbreviations: BMI, body mass index; DBP, diastolic blood pressure; FBS, fasting blood sugar; HbA1c, glycated hemoglobin; HDL, high density lipoprotein; LDL, low density lipoprotein; nHDL, non-high density lipoprotein; PPBS, post- prandial blood sugar; SBP, systolic blood pressure; VLDL, very low density lipoprotein.

Table 2 Special parameters

\begin{tabular}{|l|l|l|l|l|}
\hline Parameters & Group $1(\boldsymbol{n}=\mathbf{7 0})$ & Group 2 $(\boldsymbol{n}=70)$ & Group 3 $(\boldsymbol{n}=\mathbf{7 0})$ & $p$-Value \\
\hline Sirtuin1 $(\mathrm{ng} / \mathrm{mL})$ & $46.76(12.4-97)$ & $34.74(25.08-53.2)$ & $49.6(33.71-101.63)$ & 0.002 \\
\hline Serum fluoride $(\mathrm{ppm})$ & $0.66(0.62-0.72)$ & $0.24(0.2-0.5)$ & $0.6(0.56-0.68)$ & $<0.001$ \\
\hline Urine fluoride $(\mathrm{ppm})$ & $0.89(0.55-1.49)$ & $0.24(0.16-0.41)$ & $0.72(0.53-1.02)$ & $<0.001$ \\
\hline CML $(\mathrm{ng} / \mathrm{mL})$ & $899(625.25-1,306.5)$ & $1,815(1,100-2,591.13)$ & $1,870(1,155.1-2,272.5)$ & $<0.001$ \\
\hline
\end{tabular}

Abbreviations: CML, carboxymethyl lysine; ppm, parts per million.

Table 3 Extended diabetic profile

\begin{tabular}{|l|l|l|l|l|}
\hline Parameters & Group $\mathbf{1}(\boldsymbol{n}=\mathbf{7 0})$ & Group $2(\boldsymbol{n}=\mathbf{7 0})$ & Group $\mathbf{3}(\boldsymbol{n}=\mathbf{7 0})$ & $p$-Value \\
\hline Fructosamine $(\mathrm{ng} / \mathrm{mL})$ & $100.2(55.8-172.4)$ & $245.93(0.16-0.41)$ & $329.9(131.42-88.2)$ & $<0.001$ \\
\hline Insulin $(\mu \mathrm{IU} / \mathrm{mL})$ & $9.9(6.22-14.4)$ & $8.82(5.14-13.32)$ & $9.11(6.35-15.62)$ & 0.370 \\
\hline HOMA-IR & $2.34(1.3-3.2)$ & $3(1.99-5.62)$ & $4.35(2.4-7.1)$ & $<0.001$ \\
\hline QUICKI & $0.34 \pm 0.02$ & $0.32 \pm 0.03$ & $0.3(0.29-0.34)$ & $<0.001$ \\
\hline
\end{tabular}

Abbreviations: HOMA-IR, homeostasis model assessment-insulin resistance; QUICKI, quantitative insulin check index.

disastrous activity in a system. This study involves a very important dimension in metabolic disturbance, namely type-2 DM and fluorosis. Though fluoride is not the only important causative, fluoride shall be considered one of the triggering factors or accelerators to end up in complication.
In the present study, - Table 1 represents the demographic details of all the study patients with control group (group 1) consisting of 39 females and 31 males and DN group (group 2) with 23 females and 47 males, indicating male gender is more affected with diabetic complication 
Table 4 Extended renal profile

\begin{tabular}{|l|l|l|l|l|}
\hline Parameters & Group 1 $(\boldsymbol{n}=70)$ & Group 2 $(\boldsymbol{n}=70)$ & Group 3 $(\boldsymbol{n}=\mathbf{7 0})$ & $p$-Value \\
\hline Cystatin C $(\mathrm{mg} / \mathrm{L})$ & $0.9(0.69-1.2)$ & $4.3(3-6.5)$ & $2.3(1.46-3.69)$ & $<0.001$ \\
\hline eGFR $\left(\mathrm{mL} / \mathrm{min} / 1.73 \mathrm{~m}^{2}\right)$ & \multicolumn{5}{|l|}{} & \\
\hline CKD-EPI 2009 & $109.5(99-116.25)$ & $26(17.75-37.25)$ & $106(93-115.5)$ & $<0.001$ \\
\hline CKD-EPI 2012 & $92.5(74-118.5)$ & $60.5(40-91)$ & $118.5(95.75-137.25)$ & $<0.001$ \\
\hline
\end{tabular}

Abbreviation: eGFR, estimated glomerular filtration rate; CKD-EPI, chronic kidney disease-epidemiology.

${ }^{a}$ Equation based on creatinine.

${ }^{\mathrm{b}}$ Equation based on creatinine and cystatin $\mathrm{C}$.

Table 5 Correlation of serum fluoride and CML with sirtuin 1

\begin{tabular}{|l|l|l|l|}
\hline \multirow{2}{*}{ Parameters } & Group 1 $(\boldsymbol{n}=70)$ & Group 2 $(\boldsymbol{n}=70)$ & Group 3 $(\boldsymbol{n}=70)$ \\
\cline { 2 - 4 } & \multicolumn{2}{l|}{ Spearman's rho $(\rho)$ correlation } & \multicolumn{2}{l|}{} \\
\hline Serum fluoride $(\mathrm{ppm})$ & 0.061 & 0.292 & 0.005 \\
\hline $\mathrm{CML}(\mathrm{ng} / \mathrm{mL})$ & 0.188 & 0.054 & 0.153 \\
\hline
\end{tabular}

Abbreviations: CML, carboxymethyl lysine; ppm, parts per million.

Table 6 Correlation of HOMA-IR and QUICKI with sirtuin1

\begin{tabular}{|l|l|l|l|}
\hline \multirow{2}{*}{ Parameters } & Group 1 $(\boldsymbol{n}=70)$ & Group 2 $(\boldsymbol{n}=70)$ & Group $3(\boldsymbol{n}=70)$ \\
\cline { 2 - 4 } & Spearman's rho $(\rho)$ correlation & +0.073 \\
\hline HOMA-IR & 0.028 & +0.047 & +0.022 \\
\hline QUICKI & +0.098 & 0.004 & \\
\hline
\end{tabular}

Abbreviations: HOMA IR, homeostasis model assessment-insulin resistance; QUICKI, quantitative insulin sensitivity check index.

than females. Group 3 (type-2 DM) patients consisted of 33 females and 37 males. The mean age of the controls and cases were significantly different since onset of type-2 DM and its complications are observed during the later stages of life, and hence the mean age cases with DN (group 2) was 14 years more than group 1 (56. $04 \pm 8.2$ and $42.71 \pm 9.2$, respectively). Prevalence of diabetes by age is well represented in the IDF Diabetes Atlas ninth edition that it increases gradually from younger age (20-24 years) to adults (44-62 years) to old age (64-79 years). ${ }^{21}$ As far as BMI is considered, since the study was performed in a rural area and the staple food being finger millets (ragi), a food with low glycemic index, most of the patients' BMI was well within range, though there was some significant difference in the mean values, especially between the controls $(24.1 \pm 3.14)$ and $\mathrm{DN}(22.6 \pm 1.6)$ due to restriction in diet.

Type-2 DM is diagnosed initially by fasting blood glucose (FBS) levels with more than $110 \mathrm{mg} / \mathrm{dL}$ are considered to be hyperglycemic according to WHO and American Diabetic Association (ADA) which is found true in case of basic diabetic profile in current study with FBS of groups 2 and 3 to be $173.6 \pm 64.02$ and $182.3 \pm 67.7$, respectively, unlike the group 1 FBS $(93.94 \pm 9.4))^{1,22}$ The cut-off range of glycated hemoglobin (HbA1c) by ADA to call it as diabetes is $\geq 6.5 \%$ which was satisfied by cases groups in this study with HbA1 values of DN and type-2 DM to be $8.2 \pm 2$ and $9.2 \pm 2.4$, respectively. ${ }^{22}$

As a concluding remark, the basic tests performed proved to be confirmatory test to classify patients as diabetic cases for this study. According to KDIGO 2020 guidelines, chronic kidney disease (CKD) is defined as "persistent renal damage or albuminuria for more than 3 months diagnosed by performing eGFR and/or urine albumin estimation" or otherwise urine albumin by creatinine ration (UACR) is called as CKD. ${ }^{23}$

Current study is concentrated on the following essential parameters for renal function: serum creatinine, blood urea, and serum albumin. Among the basic renal parameters estimated, urea and creatinine $(69.03 \pm 27.5$ and $3.4 \pm 1.5 \mathrm{mg} / \mathrm{dL}$, respectively) values showed a sharp increase and albumin value $(2.8 \pm 0.8 \mathrm{mg} / \mathrm{dL})$ was declined in group 2 which is a clear sign of renal function damage coinciding with the recommendations of KDIGO $2020 .^{23}$

Lipid profile test tabulated in - Table 1 and found that total cholesterol, HDL, LDL, VLDL, and nHDL were low in group 2 than the other groups. There are many controversial studies on lipid levels in DN, Palazhy and Viswanathan and Chen et al documented that lipid molecules are elevated in severe DN cases. ${ }^{24,25}$ On the other hand, group 3 (type-2 DM) patients showed dyslipidemia indicating disturbances in lipid metabolism due to insulin resistance or poor diet control which, if continued, may lead to comorbid conditions in accordance with Wu and Parhofer. ${ }^{26}$

Eventually, regular check on lipid profile in diabetes is mandatory to avoid further complications. Special parameters considered in this study are, sirtuin $1, \mathrm{CML}$, urine, and serum fluoride. - Table 2 describes a decrease in sirtuin1, serum, and urine fluoride of group 2 (34.74 [25.08-53.2], 
0.24 [0.2-0.5], and 0.24 [0.16-0.41]) compared with other groups. In a study conducted by Gok et al in Turkey population, stated that sirtuin 1 can be a potential biomarker in type-2 DM diagnosis along with microRNA 181a and 132 which affects sirtuin1 physiological activity. ${ }^{27}$ Serum fluoride levels are high in groups 1 and 3 (0.66 [0.62-0.72] and 0.6 [0.56-0.68]) and, likewise, its excretion is also proportionate (0.89 [0.55-1.49] and 0.72 [0.53-1.02]) unlike group 2 whose serum and urine fluoride levels (0.24 [0.2-0.5] and 0.24 [0.16-0.41]) are equal, which means renal clearance is decreased. From the total fluoride ingested, in children, $80 \%$ is absorbed by mineralized tissues and 30 to $50 \%$ is absorbed in young and middle-aged people. ${ }^{28}$ Unabsorbed fluoride is mainly excreted through urine in a healthy person. ${ }^{28}$

During renal damage, fluoride excretion is hindered leading to increased serum fluoride and complications as evident from this study. In an animal study by Suzuki and Bartlett, increase in sirtuin 1 acted as a protective molecule against fluoride damage by activating autophagy which gives a new hypothesis to be applied in human biology. ${ }^{29}$ Similarly, CML values were almost equal in group $2(1,815$ [1,100-2,591.13]) compared with group $3(1,870$ [1,155.1-2,272.5]) but is decreased in group 1 (899 [625.25-1,306.5]). As described by Hammes et al in 1999, CML influences on renal function in maintaining AGE homeostasis, and CML is usually found increased in diabetes and its complications especially in $\mathrm{DN}$, and hence the finding in this study is evident of the same. ${ }^{30}$ Although, the urinary CML excretion is uncertain and not much associated with intake and the status of the individual's metabolism. ${ }^{31}$ To summarize, increased CML and fluoride act as prooxidant, restricting the effect of sirtuin 1 on cellular damage causing further complication, such as increased insulin resistance and decreased insulin sensitivity, which is in line with the findings of Uribarri et al. ${ }^{14}$

Extended diabetic and renal profile were included to assess in-depth consequences of fluoride and CML (type-2 $\mathrm{DM}$ ) in prognosis of disorder. Analogous to HbA1c glycated albumin (fructosamine) is considered to be short-term (2-3 weeks of half-life) indicator of protein glycation, an upcoming parameter of interest, its increase is directly proportional to blood glucose. ${ }^{15}$ Nevertheless, Cohen et al documented that HbA1c and fructosamine have glycosylation gap but are good predictor of average glucose of longand short-term controls which is proven right from this study from - Table 3. There is an increased fructosamine level in group 2 (245.93 [0.16-0.41]) and group 3 (329.9 [131.42-88.2]) when compared with group $1 .^{32}$ Since fructosamine levels are dependent on serum albumin concentration and eventually the serum albumin is decreased in DN, there is a fall in fructosamine concentration in DN than in type-2 DM. Fasting insulin was analyzed to calculate HOMAIR and QUICKI. Insulin values were decreased in DN and high in type-2 DM; there is no much significance in insulin values since it has a broad reference range. As we move down the table, HOMA-IR was calculated using online calculator with the formula derived by Matthews et al. ${ }^{19}$ HOMA-IR value of $\geq$ 3.5 is considered as insulin resistance as per IDF. ${ }^{33}$ QUICKI value of $<0.32$ indicates diabetes with decreasing insulin sensitivity concordant with values of Chen et al. Therefore, it is evident from previous studies and current study that HOMA-IR and QUICKI can act as better surrogate markers of insulin action in diagnosis of diabetes.

Serum creatinine is considered the gold-standard marker for diagnosis of renal impairment; however, due to least specificity and other dependent factors, there was a need for more specific biomarker and hence emerged Cys $\mathrm{C}$ which is found high in serum during renal insufficiency and specific to renal function. From - Table 4, the values of serum Cys $C$ were high in group 2 patients (4.3 [3-6.5]) than in other groups which is in agreement with study by Jeon et al. ${ }^{34}$ This study employed eGFR equations by creatinine and a combination of creatinine and Cys $\mathrm{C}$ in which group 2 showed a decline in both the equations of eGFR (26 [17.75-37.25] and 60.5 [40-91], respectively), confirming the deterioration of renal function.

Moving across the table, eGFR of groups 1 and 3 was within normal range and were not significant between both for eGFR creatinine values unlike the creatinine and Cys $C$ values which was less in group 1 (92.5 [74-118.5]) than group 3 (118.5 [95.75-137.25]) being significant in comparison with controls may be because of some outlier of Cys C values. Hence, concluding remark regarding eGFR, eGFR Eq. 2009 is a better indicator and serum Cys C alone is a better surrogate marker than creatinine in diagnosis of DN.

In the present study, the vital parameters, such as CML, fluoride, sirtuin1, Cys C, and insulin resistance and sensitivity, were compared conclude the study findings. - Table 5 serum fluoride, CML, and Cys $\mathrm{C}$ with sirtuin 1 found that serum fluoride and CML, which are the causative molecules for worsening the disease progression, were found negatively correlated in all the groups, indicating the activation of sirtuin 1 action on cells under damage. CML decrease during increased sirtuin 1 indicates deacetylation of the protein suppression, as demonstrated by Uribarri et al. ${ }^{14}$ Similarly, increased sirtuin 1 protects cells from fluoride induced stress by activating autophagy, as demonstrated by Suzuki and Bartlett in an animal study. ${ }^{29}$

Other important component of this study is insulin action (insulin resistance [IR] and insulin sensitivity [IS]) derived by HOMA-IR and QUICKI calculation. Comparison of insulin sensitivity and resistance with insulin resistance may indirectly indicate the cellular status in insulin reception. From - Table 6, negative correlation indicates decrease in HOMA-IR values, indicating decreased resistance during increased sirtuin 1 values and vice versa in regard with QUICKI, since increased QUICKI value in general means increased sensitivity. Therefore, correlation outcome of this study clearly indicates that though there is no significant correlation, the trend is an evidence for curative nature of sirtuin 1 in insulin sensing of cells for glucose metabolism not only in DM but also in other insulin-related disorders such as metabolic syndrome, obesity, thyroidism, and others. Uribarri et al correlated CML as contributor to increased IR and suppression of these shall help activating sirtuin 1 protective mechanism and revert the changes to normal which gives a lead to conclude this study. ${ }^{14}$

The present study is conducted in fluoride endemic area and recruited type-2 DM and DN cases, a correlation of fluoride exposure and excretion with eGFR were correlated 


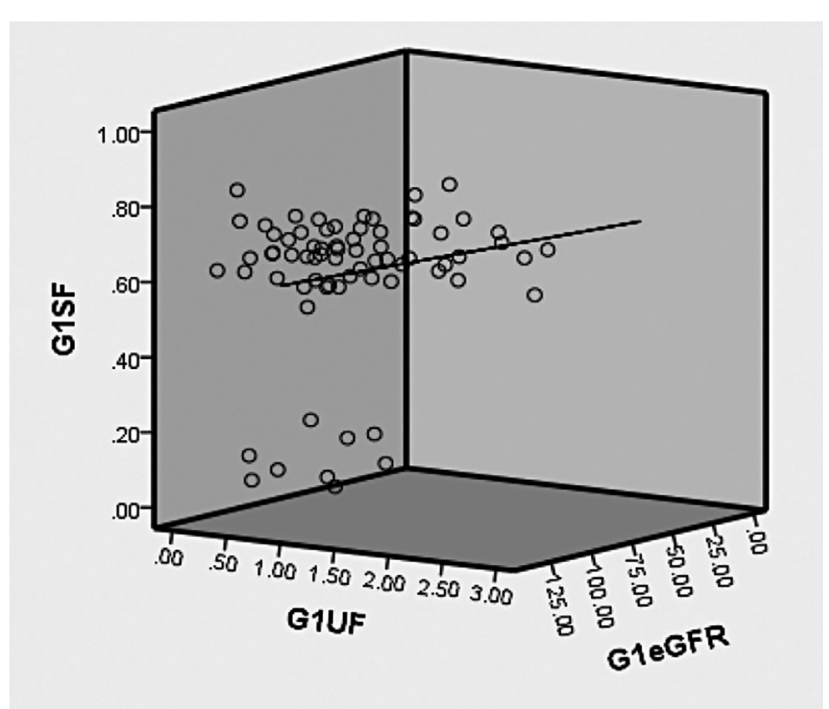

Fig. 1 Correlation of serum and urine fluoride with eGFR in group 1 (healthy controls). G1SF, group 1 serum fluoride; G1UF, group 1 urine fluoride; G1eGFR, group 1 estimated glomerular filtration rate.

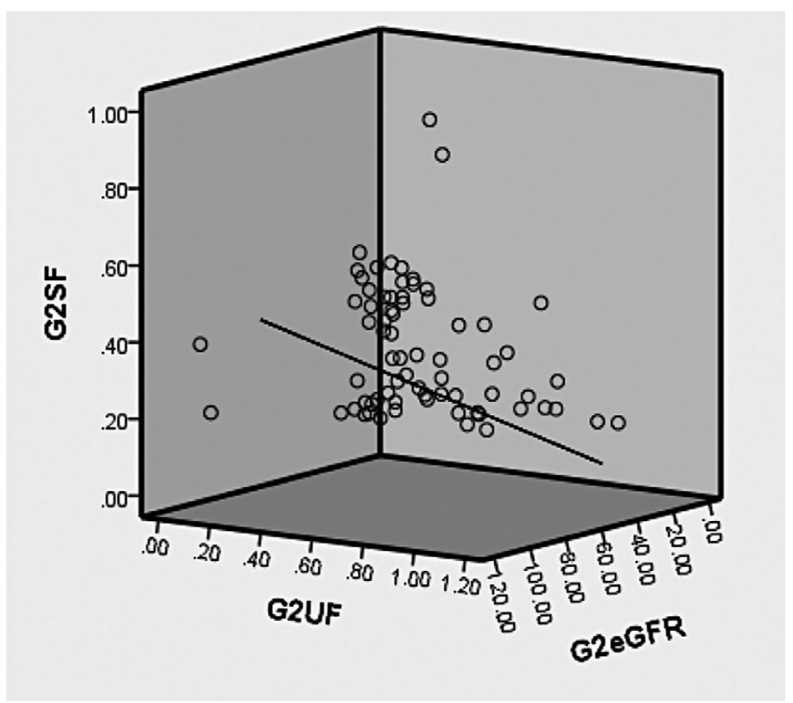

Fig. 2 Correlation of serum and urine fluoride with eGFR in group 2 (diabetic nephropathy). G2SF, group 2 serum fluoride; G2UF, group 2 urine fluoride; G2eGFR, group 2 estimated glomerular filtration rate.

in a three-dimensional graph for groups 1 to 3 (triple axis). Group 1 (controls) showed a better positive correlation indicating a normal functioning renal apparatus represented in - Fig. 1. - Fig. 2 represented a strong inverse correlation indicating decline in renal function in group 2 (diabetic nephropathy). A poor positive correlation was observed in group 3 -Fig. $\mathbf{3}$, conclusive that there may be a strong possibility of diabetic complication, especially renal impairment at the earliest.

\section{Conclusion}

From the present study, it is comprehensible that fluoride and diabetes in combination is a fast destructing disorder of normal metabolism. Since fluorosis and diabetes are globally prevailing epidemiological hitch, there is a need of a bio-

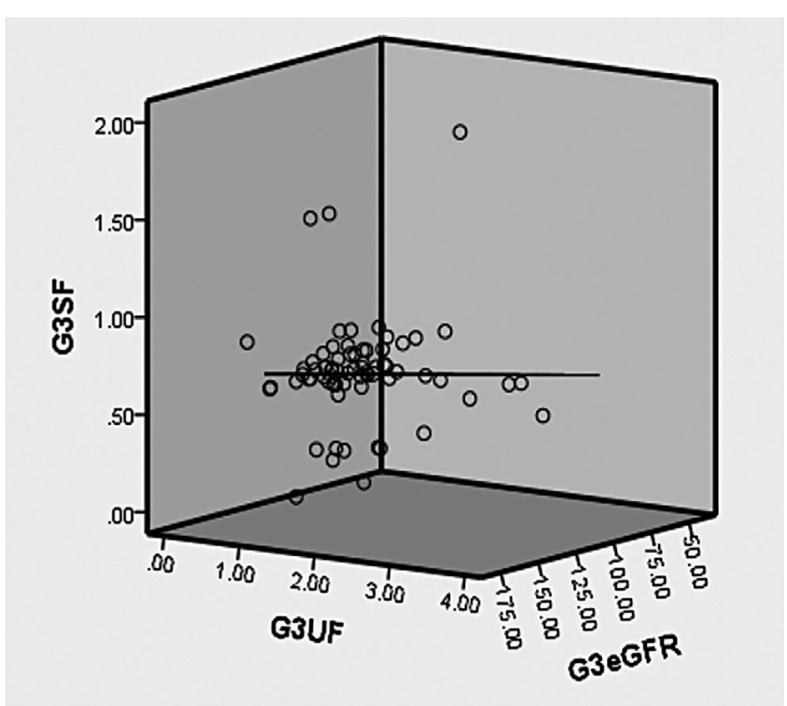

Fig. 3 Correlation of serum and urine fluoride with eGFR in group 3 (type-2 DM). DM, diabetes mellitus; G3SF, group 3 serum fluoride; G3UF, group 3 urine fluoride; G3eGFR, group 3 estimated glomerular filtration rate.

marker and/or a therapeutic molecule to trace or heal the consequences. The prime molecule of interest in this study is sirtuin 1 that is now gaining momentum in the era of aging disorders, hence it is included in this study to find its trend in diabetes and fluorosis.

The alterations in serum sirtuin 1 levels indicate the severity of damage due to stress during hyperglycemia and toxicity in fluorosis, hence sirtuin 1 can be considered a biomarker of aging. Subsequently, the correlation of CML, eGFR, and fluoride with sirtuin 1 indicates that increasing sirtuin 1 may defend the forthcoming damage, hence could be considered in therapeutics. It is clear that fluoride and CML can alter sirtuin1 values, therefore decrease in sirtuin 1 value is a consequence of hyperglycemia and fluorosis. To certain extent of injury, sirtuin 1 synthesis will be triggered, beyond which the inability will persist due to loss of normal cell function.

We could document that fluoride and CML alter sirtuin1 values. Decrease in sirtuin 1 value is a consequence of uncontrolled hyperglycemia and chronic fluorosis in DN. The levels of sirtuin 1 are directly proportional to its synthesis in $\mathrm{DM}$ and inversely proportional in DN.

To substantiate our findings and before giving a status to the molecules, sirtuin 1 and fluoride, we propose in vitro studies by using cell lines and to decide on therapeutic applications of sirtuin1. Further in vitro studies and studies comparing the nutrition intake may help find the actual point of action of molecules to find remedies to fight against aging and its effects.

\section{Authors' Contributions}

S.D.R. contributed in sample collection and analysis, data compilation and analysis. S.K.N. contributed in study design and protocol, article review, and reagent recruitment. R.A. contributed in patient selection and recruitment, treatment advice to patients, and diagnosis. M.C. contributed in field study and selection of cases from rural areas, data analysis, and results interpretation. 


\section{Statement of Ethics}

After explaining the whole procedure in patients' understandable language and providing him/her with the patient information sheet, informed written consent following the Declaration of Helsinki was obtained from the study patients, which was approved by Central Ethics Committee No: SDUAHER/KLR/CEC/35/2018-19, dated: May 14, 2018.

\section{Conflict of Interest}

Authors have no conflicts of interest to declare.

\section{Acknowledgment}

Our sincere thanks to the academy for their constant encouragement and infrastructure support. We would like to thank the laboratory technicians for sample analysis and the biostatistician for sample size calculation.

\section{References}

1 World Health Organization. Classification of Diabetes Mellitus, 2019. Geneva, Switzerland: World Health Organization; 2019

2 Mathers CD, Loncar D. Projections of global mortality and burden of disease from 2002 to 2030. PLoS Med 2006;3(11):e442

3 Ramachandran A, Snehalatha C, Shetty AS, Nanditha A. Trends in prevalence of diabetes in Asian countries. World J Diabetes 2012;3 (06):110-117

4 Verma R, Khanna P, Mehta B. National programme on prevention and control of diabetes in India: need to focus. Australas Med J 2012;5(06):310-315

5 Muninarayana C, Balachandra G, Hiremath SG, Iyengar K, Anil NS. Prevalence and awareness regarding diabetes mellitus in rural Tamaka, Kolar. Int J Diabetes Dev Ctries 2010;30(01):18-21

6 Verma A, Shetty BK, Guddattu V, Chourasia MK, Pundir P. High prevalence of dental fluorosis among adolescents is a growing concern: a school based cross-sectional study from Southern India. Environ Health Prev Med 2017;22(01):17

7 Wang Z, Jiang Y, Liu Net al.Advanced glycation end-product NEcarboxymethyl-lysine accelerates progression of atherosclerotic calcification in diabetes. Atherosclerosis 2012;221(02):387-396

8 Kim S, Benguria A, Lai CY, Jazwinski SM. Modulation of life-span by histone deacetylase genes in Saccharomyces cerevisiae. Mol Biol Cell 1999;10(10):3125-3136

9 Kaeberlein M, McVey M, Guarente L. The SIR2/3/4 complex and SIR2 alone promote longevity in Saccharomyces cerevisiae by two different mechanisms. Genes Dev 1999;13(19):2570-2580

10 Mooyaart AL. Genetic associations in diabetic nephropathy. Clin Exp Nephrol 2014;18(02):197-200

11 Brownlee M, Cerami A, Vlassara H. Advanced glycosylation end products in tissue and the biochemical basis of diabetic complications. N Engl J Med 1988;318(20):1315-1321

12 Brownlee M. Advanced protein glycosylation in diabetes and aging. Annu Rev Med 1995;46:223-234

13 Zbroch E, Bazyluk A, Malyszko Jet al.The serum concentration of anti-aging proteins, sirtuin 1 and $\alpha$ klotho in patients with endstage kidney disease on maintenance hemodialysis. Clin Interv Aging 2020;15:387-393

14 Uribarri J, Cai W, Ramdas Met al.Restriction of advanced glycation end products improves insulin resistance in human type 2 diabetes: potential role of AGER1 and SIRT1. Diabetes Care 2011;34(07):1610-1616

15 Averna MR, Carroccio A, Barbagallo CM, Montalto G, Soresi M, Notarbartolo A. Diagnostic use of fructosamine assay in the control of type II diabetes mellitus. Acta Diabetol Lat 1988;25 (01):63-68
16 Hall A, Håkansson K, Mason RW, Grubb A, Abrahamson M. Structural basis for the biological specificity of cystatin C. Identification of leucine 9 in the $\mathrm{N}$-terminal binding region as a selectivity-conferring residue in the inhibition of mammalian cysteine peptidases. J Biol Chem 1995;270(10):5115-5121

17 Knopfholz J, Disserol CC, Pierin AJet al.Validation of the friedewald formula in patients with metabolic syndrome. Cholesterol 2014; 2014:261878

18 Liu A, Reaven GM. Is measurement of non-HDL cholesterol an effective way to identify the metabolic syndrome? Nutr Metab Cardiovasc Dis 2013;23(11):1122-1127

19 Matthews DR, Hosker JP, Rudenski AS, Naylor BA, Treacher DF, Turner RC. Homeostasis model assessment: insulin resistance and beta-cell function from fasting plasma glucose and insulin concentrations in man. Diabetologia 1985;28(07):412-419

20 Katz A, Nambi SS, Mather Ket al.Quantitative insulin sensitivity check index: a simple, accurate method for assessing insulin sensitivity in humans. J Clin Endocrinol Metab 2000;85(07):2402-2410

21 Malanda B, Karuranga S, Saeedi P, Salpea P. International Diabetic Federation. 9th edition; 2019. Accessed July 29, 2020 at: https:// www.diabetesatlas.org/upload/resources/material/ 20200302_133351_IDFATLAS9e-final-web.pdf

22 American Diabetes Association. 2. Classification and diagnosis of diabetes: standards of medical care in diabetes-2018. Diabetes Care 2018;41(suppl 1)S13-S27

23 Kidney Disease: Improving Global Outcomes (KDIGO) Diabetes Work Group. Kidney Int KDIGO 2020 clinical practice guideline for diabetes management in chronic kidney disease. 2020;98(4, suppl):S1-S115

24 Palazhy S, Viswanathan V. Lipid abnormalities in type 2 diabetes mellitus patients with overt nephropathy. Diabetes Metab J 2017; 41(02):128-134

25 Chen HC, Guh JY, Chang JM, Hsieh MC, Shin SJ, Lai YH. Role of lipid control in diabetic nephropathy. Kidney Int Suppl 2005;67(94): S60-S62

26 Wu L, Parhofer KG. Diabetic dyslipidemia. Metabolism 2014;63 (12):1469-1479

27 Gok O, Karaali Z, Ergen A, Ekmekci SS, Abaci N. Serum sirtuin 1 protein as a potential biomarker for type 2 diabetes: increased expression of sirtuin 1 and the correlation with microRNAs. J Res Med Sci 2019;24:56

28 Whitford GM, Bawden JW, Bowen WH, Brown LJ, Ciardi JE, Clarkson TW. Strategies for improving the assessment of fluoride accumulation in body fluids and tissues. Adv Dent Res 1994;8 (01):113-115

29 Suzuki M, Bartlett JD. Sirtuin1 and autophagy protect cells from fluoride-induced cell stress. Biochim Biophys Acta 2014;1842 (02):245-255

30 Hammes HP, Alt A, Niwa Tet al.Differential accumulation of advanced glycation end products in the course of diabetic retinopathy. Diabetologia 1999;42(06):728-736

31 Somaraj V, Shenoy RP, Shenoy Panchmal G, Kumar V, Jodalli PS, Sonde L. Effect of herbal and fluoride mouth rinses on Streptococcus mutans and dental caries among 12-15-year-old school children: a randomized controlled trial. Int J Dent 2017; 2017:5654373

32 Cohen RM, Holmes YR, Chenier TC, Joiner CH. Discordance between HbA1c and fructosamine: evidence for a glycosylation gap and its relation to diabetic nephropathy. Diabetes Care 2003; 26(01):163-167

33 Zimmet P. The IDF consensus definition of the metabolic syndrome in children and adolescents. Assessed June 23, 2013 at: https://www.idf.org/e-library/consensus-statements/61-idfconsensus-definition-of-metabolic-syndrome-in-children-andadolescents.html

34 Jeon YK, Kim MR, Huh JE, et al. Cystatin C as an early biomarker of nephropathy in patients with type 2 diabetes. J Korean Med Sci 2011;26(02):258-263 